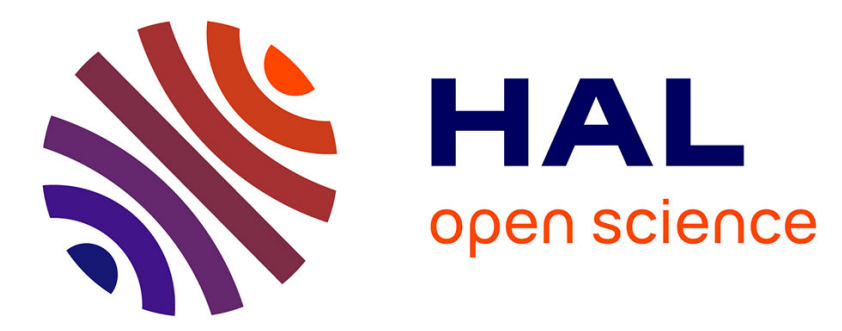

\title{
Review and comparison of different support loss models for micro-electro-mechanical systems resonators undergoing in-plane vibration
}

\author{
Benjamin Chouvion, S. Mcwilliam, A.A. Popov, C. H.J. Fox
}

\section{To cite this version:}

Benjamin Chouvion, S. Mcwilliam, A.A. Popov, C. H.J. Fox. Review and comparison of different support loss models for micro-electro-mechanical systems resonators undergoing in-plane vibration. Proceedings of the Institution of Mechanical Engineers, Part C: Journal of Mechanical Engineering Science, 2011, 226, pp.283-295. 10.1177/0954406211413351 . hal-00942174

\section{HAL Id: hal-00942174 \\ https://hal.science/hal-00942174}

Submitted on 4 Feb 2014

HAL is a multi-disciplinary open access archive for the deposit and dissemination of scientific research documents, whether they are published or not. The documents may come from teaching and research institutions in France or abroad, or from public or private research centers.
L'archive ouverte pluridisciplinaire HAL, est destinée au dépôt et à la diffusion de documents scientifiques de niveau recherche, publiés ou non, émanant des établissements d'enseignement et de recherche français ou étrangers, des laboratoires publics ou privés. 


\title{
Review and comparison of different support loss models for MEMS resonators undergoing in-plane vibration
}

\author{
B. Chouvion*, S. McWilliam, A.A. Popov, and C.H.J. Fox, \\ Materials, Mechanics and Structures Division, Faculty of Engineering, \\ University of Nottingham, Nottingham, NG7 2RD, UK.
}

May 11, 2011

\begin{abstract}
Several approaches for calculating support loss in MEMS resonators undergoing in-plane vibration are reviewed. In each of them, the support is approximated as a semi-infinite domain. The first approach is analytical and models the support as a semi-infinite thin plate. This is compared with two different finite element approaches that introduce artificial boundaries to their finite domain. In order to absorb outgoing waves and model the infinite support, a perfectly matched layer method and the use of infinite elements are considered. Simple test cases are studied and the results for the support losses predicted by the different methods are compared. It is shown that each of the methods yield similar trends. Using the developed analytical model, a parametric study is performed on the support losses of a ring-based resonator. General strategies for improving the quality factor by reducing support losses are provided.
\end{abstract}

Keywords: Support loss, anchor loss, $Q$-factor, perfectly matched layer, infinite elements.

${ }^{*}$ Corresponding author, e-mail address: benjamin.chouvion@nottingham.ac.uk, tel: +44(0) 1158467232 


\section{Introduction}

Damping is an important consideration in the design of resonant MEMS devices as their performance can be highly sensitive to the presence of damping. A variety of loss mechanisms contribute to the damping in MEMS resonators, and the required level of damping is application dependent. For example, accelerometers require significant levels of damping to ensure the vibrations are critically damped, whilst it is advantageous for vibrating rate sensors to have low levels of damping. The latter forms the motivation for the study performed here. By removing the air that surrounds the resonator, the effects of fluid damping can be eliminated. Thermoelastic damping, caused by the interaction between elastic strain and thermal effects, can be reduced by using an appropriate geometric design for the resonator [1,2]. Internal friction and surface loss are ever-present sources of energy loss in micro-resonators $[3-5]$ and can be reduced by surface treatment $[6,7]$. Support losses account for the loss of energy from the resonator through the supporting structure, and have been less widely studied compared to the other loss mechanisms. This fundamental loss mechanism forms the main focus of this study.

Practical MEMS resonators incorporate a resonator that is connected to a supporting structure. Vibration of the resonator causes the supporting structure to deform slightly, allowing some of the vibrational energy to flow from the resonator to the surrounding material. This flow arises because elastic deformations are created at the attachment point to the support, which generate stress waves. Careful design of the resonator and its at- 
tachment to the support can reduce support loss by reducing motion of the attachment point, thereby reducing the coupling between the resonator and the surrounding material [8]. Such systems may involve a mounting mechanism which isolates shear and moment reactions from the support, or the introduction of blocking masses to reduce the vibration transmitted to the boundaries [9].

To predict support loss, the coupled resonator-support system must be considered in order to understand the interaction and energy transmission between them. It is generally straightforward to model the resonator in isolation, without having to make too many assumptions, using classical finite element analysis and/or modal analysis techniques. The admittance that links velocity and applied forces at the attachment point provides a way to characterise the influence of the resonator on the support when they are modelled independently [10]. However, the disadvantage of this approach is that the physical flexibility of the attachment area is not taken into account properly in the resonator vibration. A simple and precise modelling of the interaction between resonator and support can be difficult to obtain. A more complete model that considers resonator and support together, such as for instance the one presented by Park and Park [11,12], is desirable, but the associated complexity also greatly increases, and makes it difficult to perform detailed modelling of the entire resonator-support system; so simplifications are often made. For example, the support is often assumed to be semiinfinite when calculating support losses [10-13], and this assumption will be used in this paper.

The objective of the present paper is to review and compare different 
support loss models that assume the support is semi-infinite. One of these models is used to perform a parametric study so as to gain improved understanding of the factors that influence support loss and develop strategies to minimise support loss.

In Section 2, several support loss models available in the literature are presented. For each model, convergence issues are analysed. A comparison between the different support models is given in Section 3. In Section 4, one of the models is used to calculate support loss for a ring-based resonator supported on folded-beam legs, and the influence of the leg design is considered in order to gain insights into support loss characteristics.

\section{Review of different support loss models}

\subsection{Analytical model}

Based on the fundamental work of Miller and Pursey [14], who modelled the radiation impedance of resonators on semi-infinite solids, closed-form expressions have been derived for support losses in beam resonators [13]. These expressions follow on from work undertaken by Jimbo and Itao [15]. The physical arrangement of a MEMS resonator and its support can take different forms. Typically the resonator and support are etched from the same silicon wafer and it is reasonable to assume they lie in the same plane and have the same thickness (denoted $h$ in Figure 1) [13], particularly when only in-plane vibrations are considered.

It has been proved theoretically [16] that, when the wavelength of the propagating elastic wave in the support is much larger than the size of the 


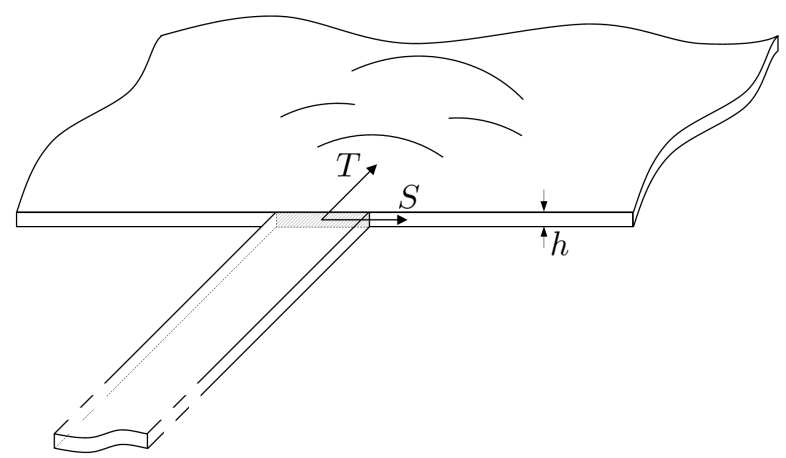

Figure 1: Semi-infinite thin plate with excitation sources.

attachment region, the coupling between the vibration modes of a MEMS resonator and the modes in its support is very weak. On this basis, a MEMS resonator and its support can reasonably be analysed separately and the stresses at the attachment region from the resonator can be considered as excitation sources for the elastic wave propagation in the support. Shear stress only was considered in [13]. However, in more complex resonators such as the ring-based resonator studied in Section 4, the supporting legs produce both shear and normal stresses simultaneously. Support excitation from normal stress was studied in $[17,18]$. Over the source region (the width of the clamped resonator), the following boundary conditions for the support are used.

- For flexural vibrations: the shear stress is assumed uniform and the normal stress is linearly dependant of the position along the width (due to the bending moment). However, the energy loss due to the bending moment has been proved to be negligible compared to that due to shear stress [18]. 
- For longitudinal vibrations, the shear stress is zero and the normal stress is uniform.

The support is modelled using the classical two-dimensional dynamic equations for a thin plate [19] subjected to the previous stress excitation sources. The attachment of the resonator to the support is assumed to be rigid initially. In other words, the deformation of the support is not considered when calculating the reactions between the resonator and support.

An analytical expression for the displacement at any point in the support is obtained by solving the governing equations of the thin-plate in the wavenumber domain. For each different boundary condition, the mean displacements (normal and tangential) along the source region, responsible for the transmitted power, are derived in order to calculate the amount of energy lost. The velocities induced by these displacements are used to determine the mean power over a single cycle, denoted $\Pi$, transmitted from the resonator to the support in each situation. This power is obtained by integrating the product of resultant forces at the attachment with the associated velocity, over one cycle of vibration, such that:

$$
\Pi=\frac{1}{2} \operatorname{Re}\left(\text { Force } \cdot \text { Velocity }^{*}\right),
$$

where Re denotes the real part, and ${ }^{*}$ the complex conjugate. The term 'Force' represents either the normal force or the shear force, and the term 'Velocity' is the associated velocity (normal or tangential direction) at the attachment area induced by the application of the corresponding force. The amount of energy lost per cycle $\Delta W$ from the support can be calculated 
explicitly as:

$$
\Delta W=\frac{2 \pi}{\omega} \Pi
$$

where $\omega$ is the frequency. The total energy lost is the sum of the energy lost from shear force $\left(\Delta W_{\text {shear }}\right)$ and normal force $\left(\Delta W_{\text {normal }}\right)$ sources. $\Delta W_{\text {shear }}$ and $\Delta W_{\text {normal }}$ are dependent on the Poisson's ratio $(\nu)$, Young's modulus $(E)$, thickness $(h)$ of the support material, and the coupling shear force $S$ and tensile force $T$ at the attachment point. From equations (2.1) and (2.2), these energy losses can be expressed as $[17,18]$ :

$$
\begin{aligned}
\Delta W_{\text {shear }} & =\alpha|S|^{2} \Psi_{s}, \\
\Delta W_{\text {normal }} & =\alpha|T|^{2} \Psi_{n},
\end{aligned}
$$

where $\alpha=4(1+\nu) /(E h(1-\nu))$, and $\Psi_{n}$ and $\Psi_{s}$ are the imaginary parts of integrals, function of $\nu$ only, which arise from the transformation of the displacement expressions from the wavenumber domain back to the frequency domain. For a Poisson's ratio of $0.28, \Psi_{n}=0.22153$ and $\Psi_{s}=0.33503$ [18]. The ratio of the maximum stored energy during a single cycle, denoted $W$, to the energy lost per cycle gives the $Q$-factor for the device, such that:

$$
Q=2 \pi \frac{W}{\Delta W_{\text {shear }}+\Delta W_{\text {normal }}}
$$

The analytical approach has its limitations. The wavelength of propagating waves must be much larger than the thickness of the vibrating structure for the two-dimensional theory to be valid. It is also unable to capture the effects of wave reflections at discontinuities in the support, since the support 
is modelled as a perfect semi-infinite thin plate. Nevertheless, because the support model is analytical and different stress sources can be considered, calculating $Q$-factors for different resonators is straightforward because it only requires the resonator model (and not the support model) to be modified. However, the main drawback is that the model is only valid under the assumptions made and results are only available for a restricted range of geometries.

In [13], the response characteristics of the resonant structure, needed to calculate the $Q$-factor (total energy and reaction forces appearing in (2.3) and $(2.5)$ ), were determined from analytical modal expansions. If the resonator geometry is complex such that modal expressions are not available, a ray tracing method $[20,21]$ can be used to obtain detailed insight into the resonator motion and energy distribution, and this approach is employed in Sections 3 and 4 .

In order to simulate the response of a semi-infinite domain, computational methods are also available, such as the use of boundary dampers, infinite elements, boundary integrals, or exact Dirichlet-to-Neumann boundary conditions (see [22-25]). The Perfectly Matched Layer method and the use of infinite elements are presented in the next sections.

\subsection{Perfectly matched layer}

The Perfectly Matched Layer (PML) technique was first introduced by Bérenger [26] for problems in electromagnetic wave propagation to model infinite boundaries. The general feature of the PML is the construction of a layer exterior to the domain of interest that damps all the waves that enter 
it from the interior domain, independent of the angle of incidence and the frequency. Waves passing through the layer are also further damped when they are reflected back, so that most of the vibration and energy entering the layer are absorbed. This layer is represented by a system of equations that are not necessarily physically realisable. Their only purpose is to prevent reflections from the outer artificial boundary. Simple "sponge layers" [27] that add dissipative terms to damp the outgoing waves were first used. However, these methods were only moderately successful because reflections were also created by gradients within the layer. To be effective, the layer must be designed so that there is no impedance mismatch to reflect waves back from the interface between the layer and the rest of the domain. The PML "perfectly matches" the impedance of the interior and exterior domains, and there are therefore no spurious reflections at the interface.

Bindel and Govindjee [28] considered the PML method for support loss calculation in MEMS resonators, and implemented the approach in a finite element code called HiQLAB [29]. When a PML is used to model infinite boundaries in a finite element code, complex mass and stiffness matrices are generated. As a consequence, complex eigenvalues $\omega$ are obtained for the governing equations and the $Q$-factor can be calculated directly using [28]:

$$
Q=\frac{|\omega|}{2 \operatorname{Im}(\omega)}
$$

where $\omega$ is the natural frequency and Im denotes the imaginary part.

Bindel and Govindjee interpreted the PML as a complex-valued change of coordinates which could be applied to any linear wave equation. The 
elements use complex stretching of the coordinate axes to introduce artificial damping to the wave. It is worth noting that the complex coordinate stretching has as a prerequisite the analytic continuation of the equations and their solutions. The coordinate transformation for a $1 \mathrm{D}$ case can be expressed in the form:

$$
\tilde{x}=x-\frac{\mathrm{i}}{k} \int_{0}^{x} \sigma(s) \mathrm{d} s
$$

where $\tilde{x}$ is the new coordinate, $x$ is the "real" axial coordinate, $\mathrm{i}=\sqrt{-1}$, and $k$ is the wavenumber. $\sigma$ is a PML attenuation function that is used to reduce the amplitude of the waves when they travel in both directions: on the way to the outer boundary, and on their way back to the resonator. As a general idea, when $\sigma$ is chosen to be zero at the boundary with the finite medium, the solution is the same for both media and hence the wave enters the PML without any reflection at the boundary. When $\sigma>0$, the wave decays in the direction of travel. Figure 2 illustrates the case of a linear attenuation function $\sigma$ with end magnitude $\beta\left(L_{p}-L\right)$, where $L$ and $L_{p}$ are respectively the length of the finite region and the PML domain. Since waves decay so rapidly in the PML region, a good approximation to the infinite domain is obtained even if the condition of zero-displacement at the outer boundary (in $L_{p}$ ) is enforced [28].

In order to correctly model a semi-infinite domain, the main objective of the PML is to minimise the waves that are reflected back in the finite domain. It should ideally absorb completely the outgoing waves and no reflection should occur. However, due to the way in which the problem is 


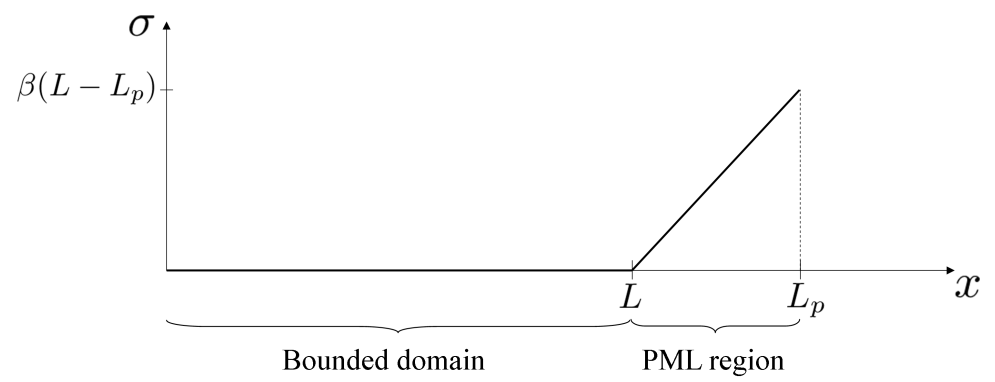

Figure 2: Linear attenuation function used in the PML method for a onedimensional wave.

discretised, some energy is always reflected back. A precise analysis of these reflections was presented by Bindel and Govindjee [28], and Koyama [30], where the total reflection due to the PML is separated into: the end termination reflection, introduced by a finite termination of the PML; the interface reflection, introduced by the discretised PML at the PML interface; and the computed reflection, due to round-off errors in computing calculations.

The different parameters that have an effect on the $Q$-factor are:

- Geometrical parameters: the dimension of the PML region $\left(L_{p}-L\right)$, and the dimension of the "finite" support region $(L)$ meshed using standard finite elements;

- Meshing parameters: the number of elements in the PML region, and the interpolation order of the elements in the PML (linear, quadratic, cubic);

- PML function properties: the end value parameter $\beta$ of the PML absorbing function $\sigma$, and the polynomial order of the PML absorbing function $\sigma$. 
The work reported in [28] and [30] provides guidelines for selecting PML parameters in a rational way and these are used as the starting point for the results presented later. Different parameter values are investigated to ensure that converged results are obtained. All of the results presented in Section 3 are obtained after the convergence study has been completed. These results indicate that the PML method is time-consuming and that the results obtained are not "exact" $Q$-values. For this reason, an alternative finite element method with infinite elements was also investigated, and is discussed next.

\subsection{Infinite elements}

Infinite boundaries can be modelled using the finite element method using specific "infinite elements". First- and second-order infinite elements based on the work of Zienkiewicz et al. [31] for static response and of Lysmer and

Kuhlemeyer [32] for dynamic response are implemented in ABAQUs ${ }^{\mathrm{TM}}$ to model infinite boundaries. The energy is artificially attenuated and lost in these elements. They are used to mesh the far-field region, in conjunction with standard finite elements, which model the area around the resonator.

The infinite elements provide a theoretically non-reflective boundary to the model by using special shape functions that eliminate artificial wave reflections from the truncated domain of the model. Regular shape functions are used to model the displacement variables $u$ while growing shape functions are used for position variables $r$ [33].

A one-dimensional case is presented next and illustrated in Figure 3. The infinite element contains three nodes, whose nodes are identified by 


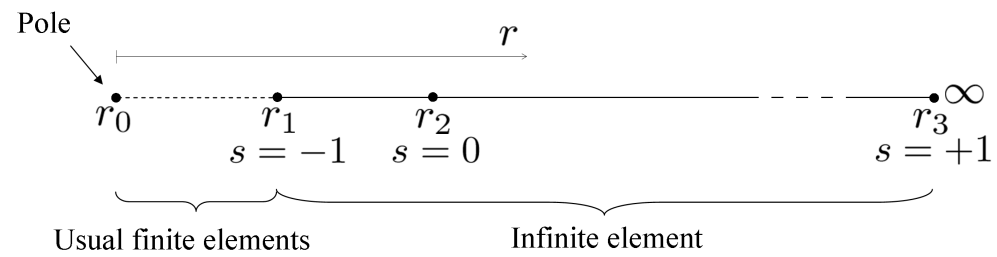

Figure 3: Nodal variable position for a one-dimensional infinite element in ABAqus $^{\mathrm{TM}}$.

$s_{i}=-1,0$ and $1(i=1,2,3) . r_{i}$ and $u_{i}$ respectively represent the distance from the "pole" that corresponds to the source of propagating waves, and the displacement of node $i$. The particular chosen shape function such that:

$$
r=\frac{-2 s}{1-s} r_{1}+\frac{1+s}{1-s} r_{2}
$$

and the following standard quadratic interpolation for the displacement:

$$
u=\frac{1}{2} s(s-1) u_{1}+\left(1-s^{2}\right) u_{2}
$$

give

$$
\begin{array}{ll}
\text { at } \mathrm{s}=-1: & r=r_{1} \quad \text { and } \mathrm{u}=\mathrm{u}_{1}, \\
\text { at } \mathrm{s}=0: & r=r_{2} \quad \text { and } \mathrm{u}=\mathrm{u}_{2}, \\
\text { at } \mathrm{s}=1: & r \rightarrow \infty \quad \text { and } \mathrm{u} \rightarrow 0 .
\end{array}
$$

Hence the position coordinate approaches infinity, and the displacement of the propagating wave decreases to zero at the outer edge of the infinite element. 
The infinite element is shown to work well for static and dynamic analyses [33]. In any case, it is assumed that the response adjacent to the boundary of the infinite elements is of sufficiently small amplitude that the medium responds in a linear elastic fashion. The infinite elements have to be placed sufficiently "far" from the pole. They also have to be arranged such that the dominant direction of wave propagation is orthogonal to the boundary, which means that the outer boundary must be semi-circular.

The fundamental in-plane mode of a simple cantilever beam is studied in ABAqus ${ }^{\mathrm{TM}}$ to check convergence using infinite elements. The beam is meshed using classical two-dimensional quadratic plane stress elements. The support is divided in two different sections: a bounded domain of length $R$, meshed with standard finite elements, and an infinite domain, meshed using the infinite elements described previously. The beam width is meshed using 4 elements, and the element size in the support gradually increases to fill the entire bounded domain. The outer ends of the infinite elements are placed at an approximate distance $2 R$ from the pole (origin of the propagating waves - the beam attachment point).

The support is supposed to be infinite and a change in the bounded domain length $R$ should theoretically not influence the results for the natural frequency and $Q$-factor. For different values of $R$, a steady state analysis is performed in the vicinity of the expected first natural frequency. The $Q$ factor is then calculated from the resulting amplitude-frequency curve and defined as:

$$
Q=\frac{\omega_{0}}{\Delta \omega}
$$




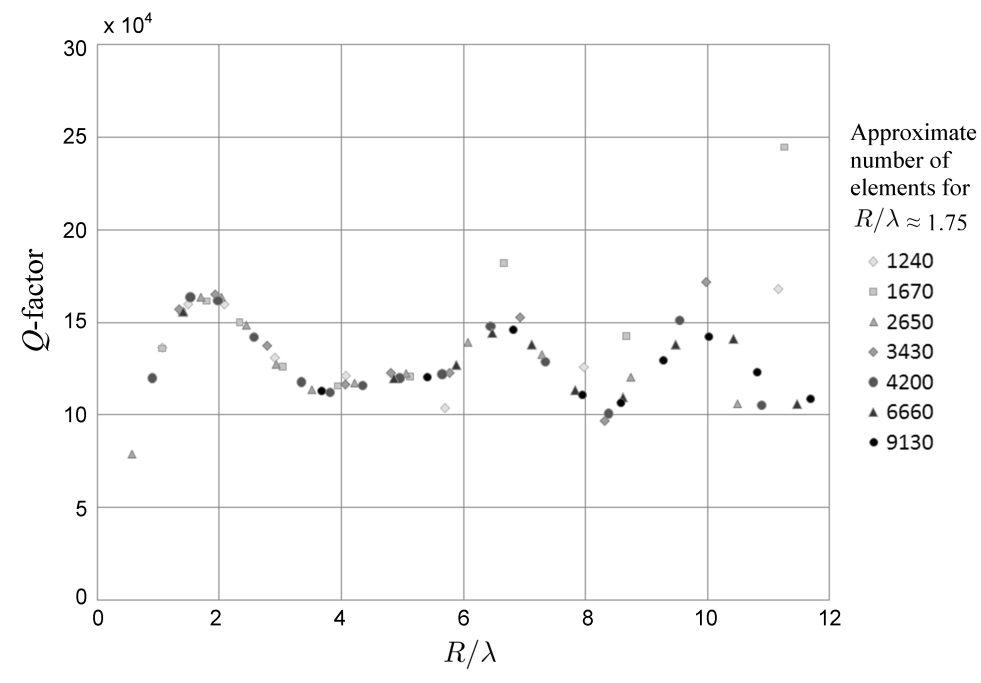

Figure 4: Convergence analysis. $Q$-factor function of $R / \lambda$ calculated for different mesh densities.

where $\omega_{0}$ is the natural frequency of interest and $\Delta \omega$ is the half-power bandwidth at $-3 \mathrm{~dB}$ of the maximum amplitude. This is the common method used to measure $Q$-factors.

It is found that the calculated $Q$-factor is actually a function of $R$, and it is expected that this dependency is also a function of the transverse wavelength $\lambda$ of the propagating shear waves in the support. The results are illustrated in Figure 4, which shows the calculated $Q$-factor for different mesh refinements and different values of $R$. With a very coarse mesh, the $Q$-value quickly diverges as $R$ increases. This is probably due to the difference in size between the bounded domain and the resonator itself. It is important to notice that, for example, when $R / \lambda \approx 10$, the bounded domain is actually 3000 times larger than the beam width. It is understandable that a coarse mesh struggles to correctly model the energy propagation and dissipation 
arising from a relatively small resonator. With a fine mesh, the $Q$-value first shows a maximum around $R / \lambda \approx 1.75$, then seems to stabilise between $3<R / \lambda<5$, and finally oscillates for $R / \lambda>6$. This simple convergence test demonstrates the complexity of calculating $Q$. Here $Q$ is highly dependent on the mesh density and on the support dimensions. However this study shows that a range of approximate values of $Q$ can still be obtained using the infinite elements. From the analysis of Figure 4 and especially the results obtained with the finest mesh, it seems sensible to use as a maximum $Q$-value, the value obtained when $R / \lambda \approx 1.75$ and as an appropriate minimum $Q$-value, the value obtained when $R / \lambda \approx 4$. These two particular values of $R / \lambda$ have been used in the studies presented in Section 3 .

\section{Comparison of the models}

The objective is to compare and validate the different support models for any resonator design. The following applications consider a wide range of possible resonator-support characteristics, in which support losses occur either from shear stress excitation, normal stress excitation, or a combination of those. Due to the complexity of creating meshes in the HIQLAB program, the difficulty to obtain a rapid converged result, and the computing cost of using finite elements methods, the methods presented in Section 2 are applied to simple test cases only.

A cantilever beam vibrating in its fundamental mode is studied first. Resonator and support are made in silicon, from the same wafer, and have identical material properties. For a fixed beam width, the calculated $Q$ - 


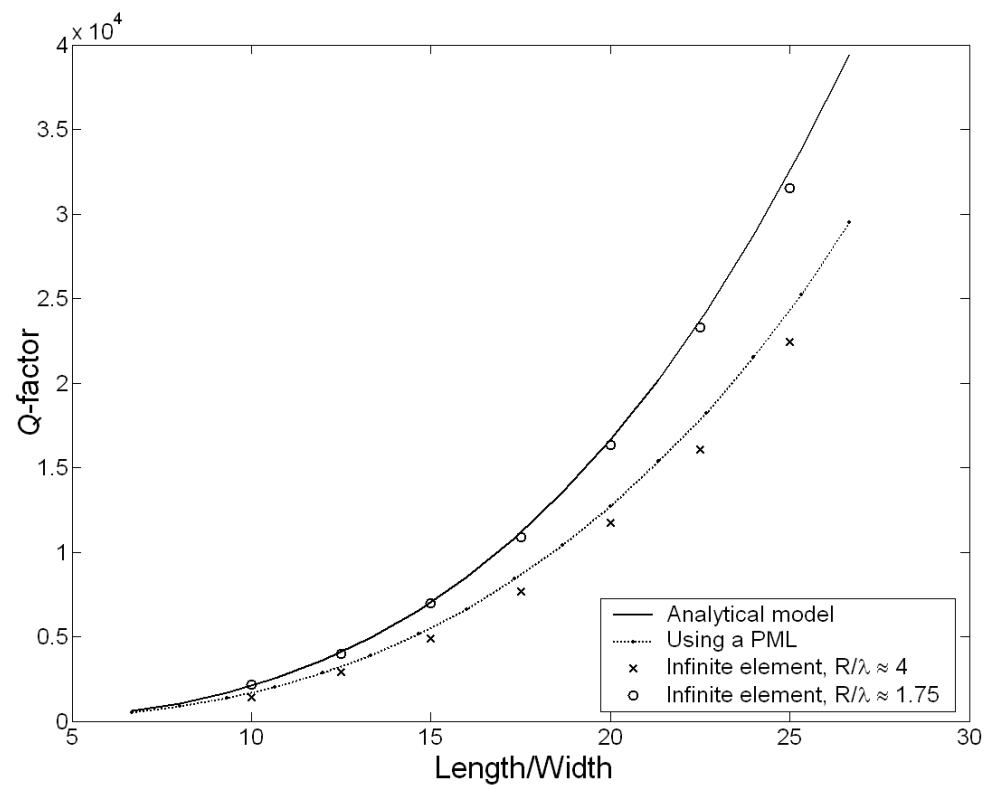

Figure 5: Cantilever beam case. $Q$-factor calculated using the three different methods as a function of the length/width ratio.

factor is plotted as a function of the length/width ratio of the beam in Figure 5. This graph compares the results obtained using the PML method in HiQLAB, infinite elements in ABAqus ${ }^{\mathrm{TM}}$, and the analytical model. In the analytical model, only shear stresses are considered at the resonatorsupport interface (normal stresses are neglected - see Section 2.1). The energy stored and clamped forces in the resonator are obtained using modal expressions. All curves show the same trend, and the results display a cubic character when the beam is made longer and/or thinner (as explained by Hao et al. in [13]). Even though the infinite element method gives a "range" of correct $Q$-values, it is shown that they are all in reasonable agreement. A possible explanation of the discrepancies is the different way the attachment is modelled. A rigid attachment assumption is considered in 
the analytical model, whereas the coupled resonator-support system, with high stress concentration in the attachment area, is modelled in the finite element approaches.

It is important to note that for more complex-shaped resonators, the attachment resonator-support may undergo flexural and longitudinal vibrations simultaneously, opposed to the simple flexural vibration of a beam resonator. For instance in a xylophone bar resonator, complex relations between the geometry and $Q$, arising from modal tuning of the component, have been found [34] using a PML method. In the analytical model presented in Section 2.1, the coupled flexural-longitudinal vibration provides a complication as the support model uses two different expressions for shear and normal stress sources. To validate the model when flexural and longitudinal vibrations occur simultaneously, another test case is studied. It consists of a two-beam system, where the beams are at right angles to each other. The first beam is attached to the infinite support, whereas the second beam (called beam ' 2 ') is free. The $Q$-factor corresponding to the first mode of vibration is studied.

For a fixed beams width, the length of beam ' 2 ' is varied. The ray tracing method [20] is used to calculate the natural frequency of the first mode, its corresponding strain energy, and shear and normal forces at the clamped boundary. The energy loss caused by the shear force and that caused by the normal force are calculated separately using (2.3) and (2.4), and then added up to obtain the overall energy loss. The objective was to take account of a wide range of resonator-support configurations that includes several complex-shaped resonator characteristics. For instance, in 


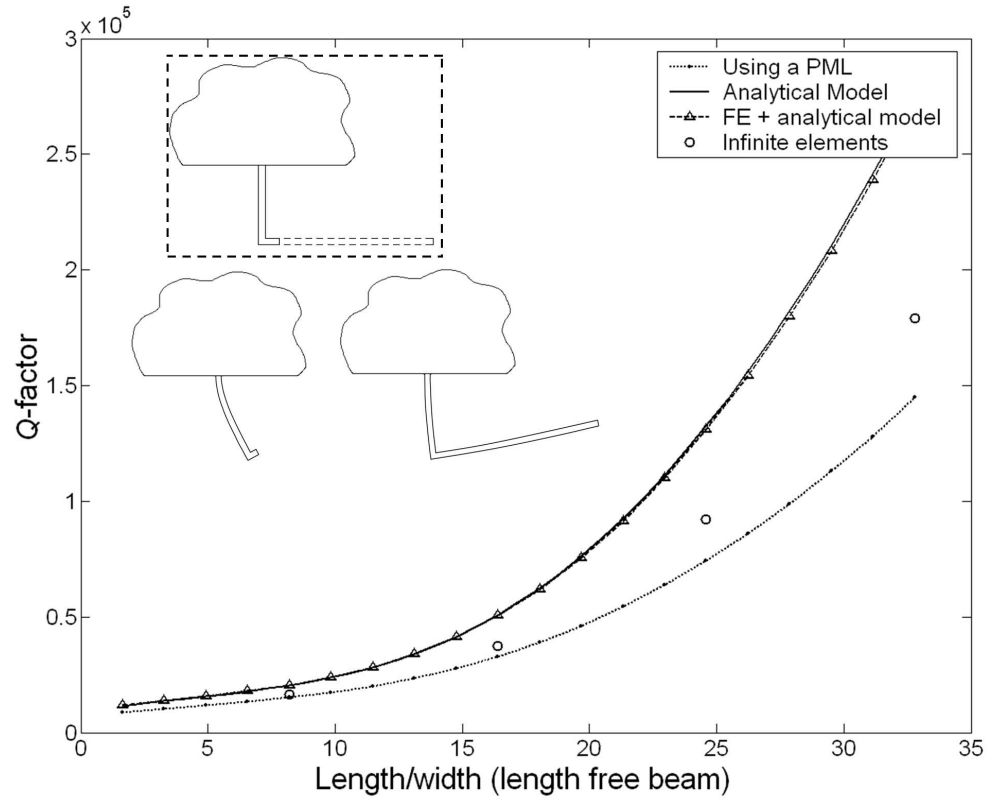

Figure 6: Two-beam case. $Q$-factor calculated using the different methods as a function of the length/width ratio of beam ' 2 '. The system studied is shown in the inset. Schematic representations of the mode shapes, for the shortest and longest beam '2' considered, are also illustrated.

the nominal design of the ring-based resonator studied in Section 4, the energy lost due to shear force is approximately 4 times larger than the one due to normal force, for the modes of interest. From the range of length/width ratio considered in the present test case, the ratio of energy lost due to shear force over the energy lost due to normal force varies between 7000 for short beam ' 2 ' and 0.3 for long beam ' 2 ' [18]; showing that this test case can represent a wide range of features.

The $Q$-factor calculated with the different methods, when the length of beam ' 2 ' is varied, is presented in Figure 6. For the infinite element method, the maximum $Q$-value, obtained when $R / \lambda \approx 1.75$, has been used. Again, 
all the curves show a similar trend. However, it appears in this case, which is not true for the simple cantilever beam study, that the relative $Q$-difference increases when the length/width ratio of beam ' 2 ' is higher. In the high length/width range, normal forces are predominant at the attachment point. Thus, a possible explanation is that the analytical support model does not properly quantify the loss occurring when normal stresses are predominant. An alternative explanation could be that the ray tracing method struggles to correctly model the beam corner when it is subjected to high stresses. The thin beam theory used in the ray tracing does not consider high stress concentrations at the corner, as only the centreline is modelled, and the complexity of a real corner could not be properly modelled.

However, a simple study has ruled out the hypothesis of inaccurate modelling by the ray tracing method. The same system has been modelled in ABAQUS $^{\mathrm{TM}}$ using quadratic elements. A modal analysis in ABAQUS ${ }^{\mathrm{TM}}$ gives the modal strain energy of the entire system and the forces at the clamped boundary for the first mode of vibration. These clamped forces and strain energy are inputted into the analytical model of the support presented in Section 2.1, and the $Q$-factor is calculated. These results are illustrated in Figure 6 . This study represents a combination between the analytical model of the support and a finite element analysis of the vibrating structure. The excellent agreement achieved indicates that the ray tracing method correctly models the vibrations, and that the difference between analytical and PML methods is only due to the support model.

In conclusion, Figures 5 and 6 indicate that the three different support models give the same range of $Q$-values and same trends when the 

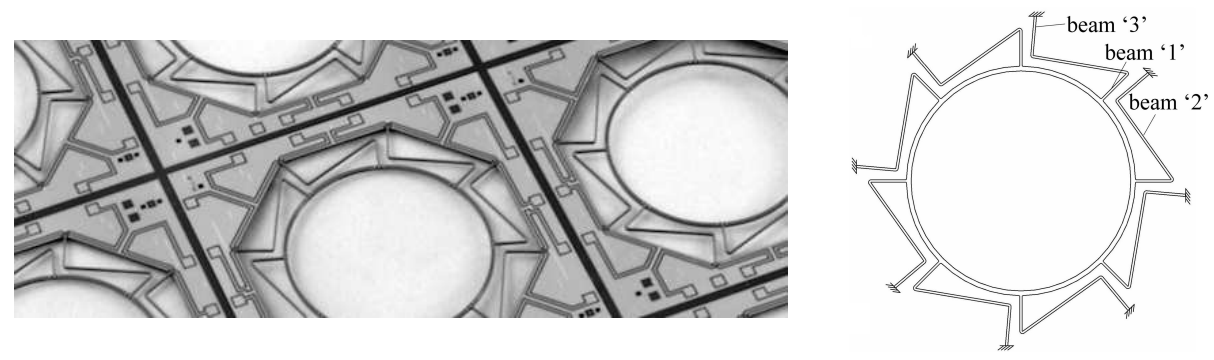

Figure 7: Photograph of a ring-based rate sensor on its silicon wafer (courtesy AIS), and schematic representation of the resonator.

length/width ratio is changed. As this study illustrates support losses when

the energy loss due to shear force is dominant, and when the energy loss due to normal force is dominant, this validates the analytical model even for complex-shaped resonator, and shows that it can be used with confidence to obtain trends of $Q$-values. The next section uses this model to study the influence of the leg design on the $Q$-factor of ring-based resonators.

\section{Influence of leg design on the $Q$-factor of a ring- based resonator}

\subsection{Nominal study}

The method referred to as the Analytical Model in Section 2 is used here to calculate the $Q$-factors of a ring-based resonator developed by Atlantic Inertial Systems (AIS). Figure 7 shows a photograph of this complex-shaped resonator on its silicon wafer and a schematic representation of the nominal device. The resonator is composed of a thin ring and eight identical uniformly-spaced legs in the form of folded beams. 


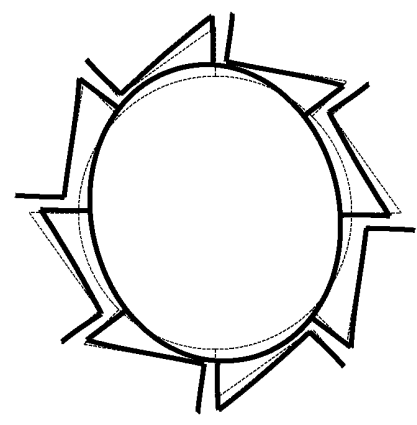

(a) $2 \theta$ mode

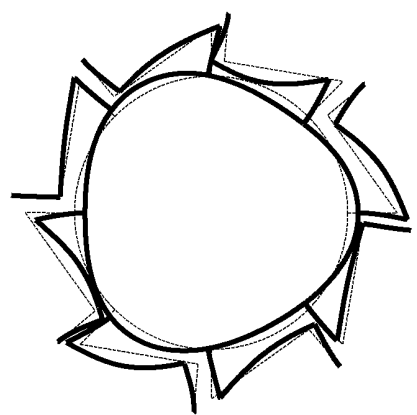

(b) $3 \theta$ mode

Figure 8: $2 \theta$ and $3 \theta$ mode shapes of the ring-based resonator with nominal dimensions.

\begin{tabular}{ccccc}
\hline & \multicolumn{3}{c}{ Damping predictions } & \multirow{2}{*}{ Measurements [35] } \\
\cline { 2 - 4 } & Support loss & Thermo. [35] & Total & \\
\hline $2 \theta$ mode & 147600 & 11731 & 10867 & 9455 \\
$3 \theta$ mode & 80660 & 17783 & 14571 & 12434 \\
\hline
\end{tabular}

Table 1: $Q$-factors predictions and measurements for the $2 \theta$ and $3 \theta$ modes.

The process employed to calculate $Q$ can be summarised as follows. Firstly, assume that the resonator is clamped and use the ray tracing approach $[20,21]$ to calculate its natural frequencies and associated mode shapes. Then, from the mode shape, calculate the total energy stored $W$ and the forces $T$ and $S$ at the clamped end. Use (2.3) and (2.4) to obtain the energy loss due to the forces $S$ and $T$, and then use (2.5) to calculate the $Q$-factor.

The nominal dimensions and material properties of the ring-based resonator are identical to those given in $[20,21]$. The $Q$-factors for the so-called $2 \theta$ and $3 \theta$ modes, whose mode shapes are illustrated in Figure 8, are calculated using the analytical method and are given in Table 1. 
These results are compared with Wong's experimental $Q$-factor measurements and theoretical predictions [35] for thermoelastic damping (denoted "Thermo." in Table 1) in similar resonators. The predicted total $Q$-factor is also shown for comparison in Table 1 and is defined as the inverse of the

total energy loss $\left(Q_{\text {Total }}=\left[\sum 1 / Q_{i}\right]^{-1}\right)$. The measured overall $Q$-factors consider all damping mechanisms. The comparison of the simulated and measured $Q$-factors gives confidence in the support loss model developed and used here. Indeed, it is interesting to note that the total energy loss predicted (inverse of the $Q$-factor) considering support loss and thermoelastic damping is only slightly smaller than the energy loss measured experimentally. It may be possible to obtain improved agreement by including damping contributions arising from surface losses and air damping in the computational model.

In applications, the excited and sensed modes are usually the $2 \theta$ mode and its orthogonal companion. Only results relating to the $2 \theta$ modes are presented in the following sections as they are of primary interest.

\subsection{Change of leg geometry}

In this section, the geometry of the leg is modified. The beam lengths and angles between the beams that make up the leg are changed. The beams are numbered as follows: beam ' 1 ' (length $L_{1}$ ) is attached to the ring, beam ' 2 ' connects beams ' 1 ' and ' 3 ', and beam ' 3 ' (length $L_{3}$ ) is clamped to the support. The beam numbering is illustrated in Figure 7. In the parametric study performed, the geometry is constrained such that: 
- Beam ' 1 ' and beam ' 3 ' are oriented radially (i.e. their centrelines pass through the centre of the ring).

- The total angle subtended by the leg, angle between beam ' 1 ' and beam ' 3 ', is equal to $40^{\circ}$. This ensures that the ring contains eight legs with a $5^{\circ}$ angular separation between each.

- The point of attachment to the support is a fixed point. This means that the distance between the clamped end and the centre of the ring is constant. The reason for this is that the modifications to the leg designs are not intended to change the dimension of the silicon wafer on which the resonators are etched nor their position on the wafer.

$L_{1}$ and $L_{3}$ are varied independently over a range defining the shortest and longest beam configurations in the allocated space (between the ring and the support). When one of these lengths varies, the other parameters (e.g. angles between them) vary accordingly by following the constraints mentioned above. For each value of the couple $\left(L_{1}, L_{3}\right)$, the $Q$-factor is calculated following the procedure described in Section 4.1.

Results for the $Q$-factors are presented in Figure 9, where it can be seen that leg designs with a longer beam ' 1 ' generally have a larger $Q$-factor. If at the same time, beam ' 3 ' is shortened, then the largest $Q$ is obtained, resulting in a significant improvement in performance. For this particular design, the $Q$-factor is approximately 3 times larger than the value for the nominal design. The associated mode shape is presented in Figure 10(a). It can be seen clearly from the mode shape that the ring contributes in an important way to the overall energy of the resonator, maximising the 


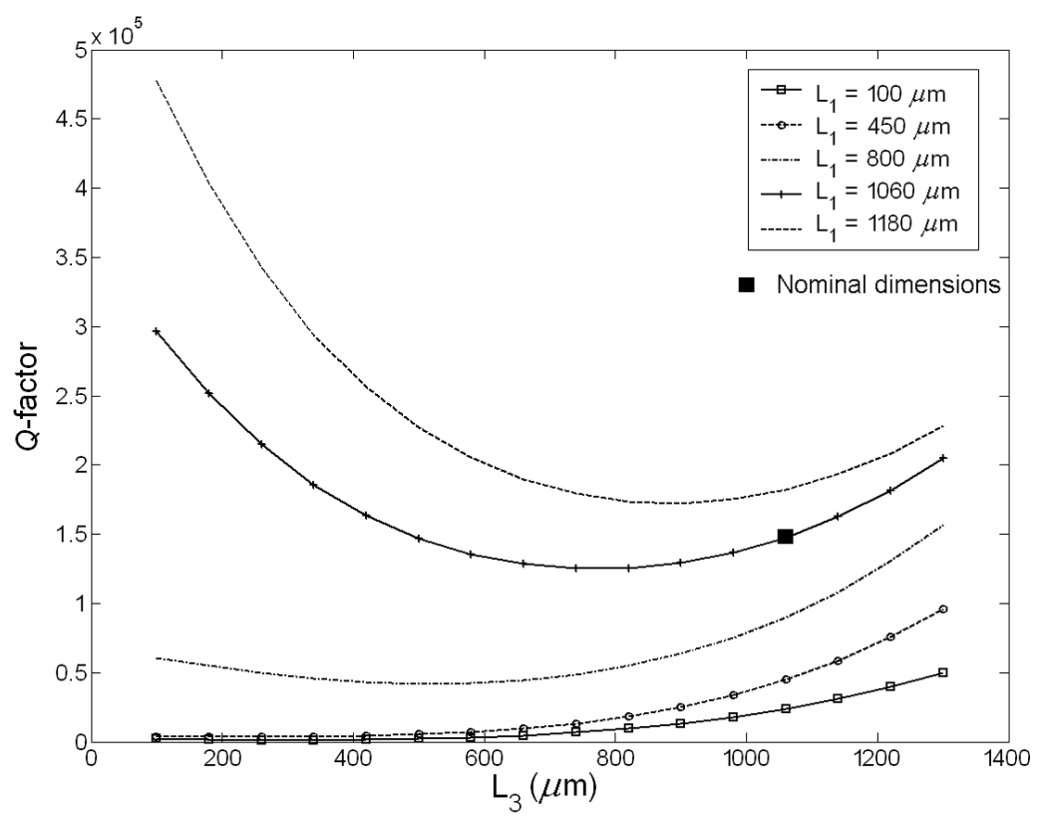

Figure 9: $Q$-factors of the $2 \theta$ mode for different values of $L_{1}$ and $L_{3}$.

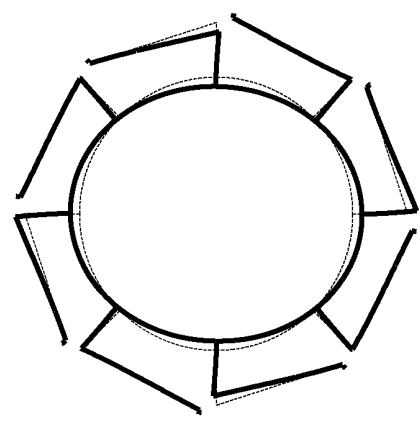

(a)

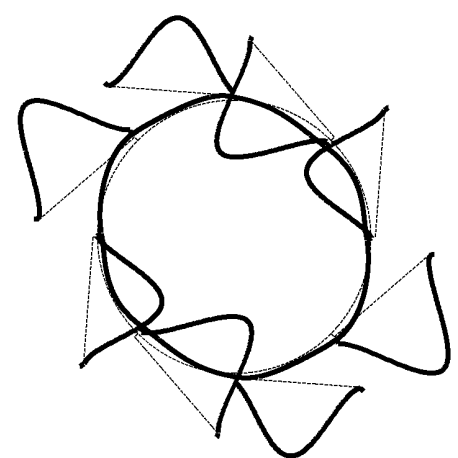

(b)

Figure 10: Deformed shape of the $2 \theta$ mode for a long beam ' 1 ' and a short beam ' 3 ' (a), and for a short beam ' 1 ' and a short beam ' 3 ' (b)

energy stored. Also, the leg is made more flexible when beam ' 1 ' is longer, i.e. the clamped end has less influence on the ring vibration. These two reasons explain the larger $Q$-value obtained. At the same time, the natural 
frequencies do not change much for the variations considered. A difference of approximately $350 \mathrm{~Hz}$ is found, which is small compared to the nominal frequency of operation $(\approx 14 \mathrm{kHz})$. It is important to control the associated change in frequency because a large variation will modify the thermoelastic loss [35].

From Figure 9, it can be seen that the design with a short beam ' 1 ' and a short beam ' 3 ', whose mode shape is illustrated in Figure 10(b), is clearly inefficient compared to the nominal design. The $Q$-factor in this case is approximately 100 times smaller than the nominal one and the $2 \theta$ frequency is greatly increased. In this case the resonator frequency almost matches one of the leg natural frequencies considered separately. For this particular design, the legs are as straight as possible within the applied constraints, and have a large influence on the ring vibration. The leg flexibility is greatly reduced and the contribution of the legs to the overall resonator deformation is increased. From the mode shape, it is clear that the ring deformation is less important compared to the legs in beam portion ' 2 ', because the leg vibrates at a frequency close to one of its own natural frequencies. This indicates that most of the energy is contained within the legs, increasing the support losses and reducing the $Q$-factor.

Looking directly at the energetic transmission and reflection coefficients at the different corners of the legs could give some general ideas and guidelines about which angles are to be used or avoided to minimise the energy propagating away through the legs. Wu and Lundberg [36] presented quantitative results on these transmission coefficients and they generally confirmed the fact that large angles facilitate the direct transmission of waves of the 
same type, whereas acute angles would tend to increase the reflection of waves. Even though their work neither takes account of the transmission and creation of evanescent terms after the discontinuity, nor takes account of the infinite reflection-transmission process that occurs between the corners, it still indicates that the angles present in the leg when beam '1' and beam '3' are short are inefficient for energy transmission. Another general guideline to minimise support losses would be to avoid designs where the natural frequency of the resonator coincides (or is in the near vicinity of) one of the leg natural frequencies, as this facilitates vibrational energy transmission.

\subsection{Change of leg width}

In this section, the geometry of the leg is maintained at the nominal design. However, the in-plane width of the leg is modified. The nominal leg and ring widths are equal to $61 \mu \mathrm{m}$ and $121 \mu \mathrm{m}$ respectively. Here, the leg width takes values between $30 \mu \mathrm{m}$ and $105 \mu \mathrm{m}$.

Results for the natural frequencies and the associated $Q$-factors as a function of leg width are shown in Figure 11. From this graph, it can be seen that the $2 \theta$ frequency increases when the leg width increases, whilst

the $Q$-factor decreases when the leg width increases. This is mainly because thin legs are more flexible in bending and constrain the ring less. This ensures that the vibration of the ring is closer to that of a ring without support, maximising the energy stored. In contrast, wide legs have a strong influence on the ring vibration and restrain its amplitude. From Figure 11, the $Q$-factor trend as a function of the leg width $b$ can be approximated to $Q \approx 1 / b^{4}$. This means that when the width of the leg is half the nominal 


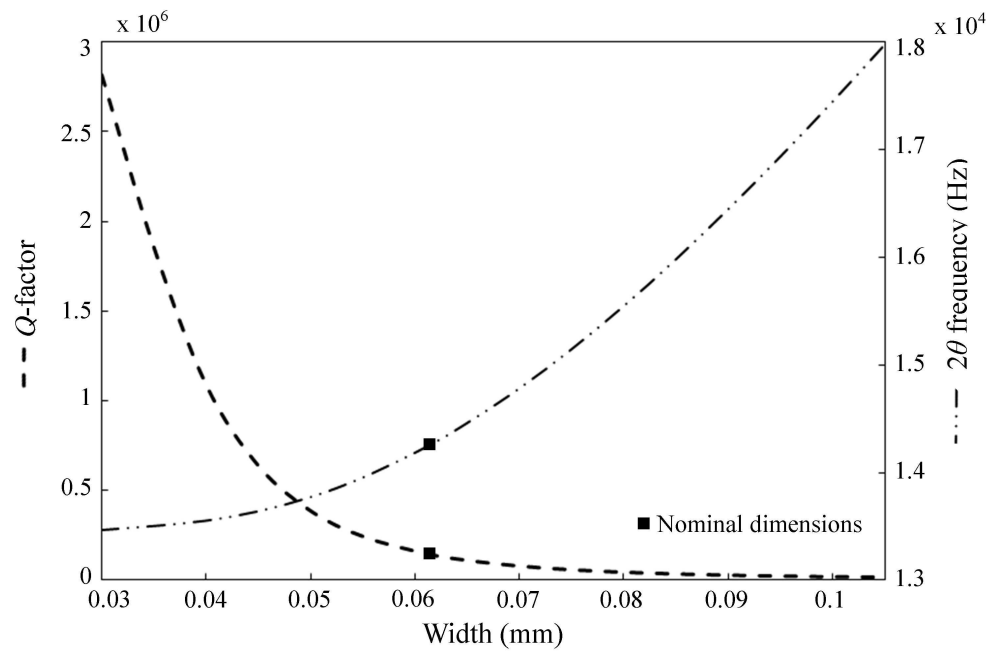

Figure 11: $2 \theta$ frequencies $(\mathrm{Hz})$ and the associated $Q$-factors function of the leg width.

value, the $Q$-factor is approximately 16 times larger than the nominal $Q$ factor. This relationship between $Q$ and $b$ can be compared to that obtained for other designs: $Q \propto 1 / b^{3}$ for a simple cantilever beam resonator [13] and $Q \propto 1 / b^{6}$ for a disk resonator supported on a single straight beam [37]. The $Q$-factor here, where the legs stay relatively flexible for any width, is therefore more sensitive to a change of width than for a cantilever beam, in which the internal stored energy in the resonator increases at the same time as the energy propagating away; but less than for a disk resonator, in which a larger supporting beam clearly increases its stiffness, and has more influence on the disk vibration.

In order to better understand the effect of the width change on the resonator vibration characteristics, it is interesting to consider the energy distribution within the resonator as a function of the leg width. Figure 12 


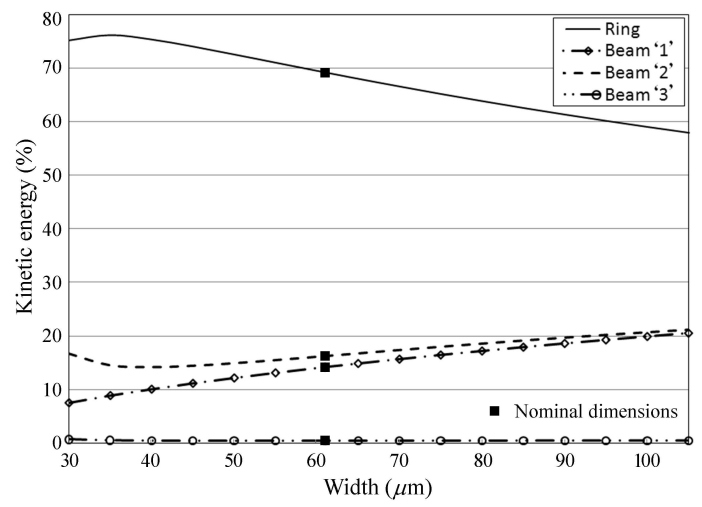

(a)

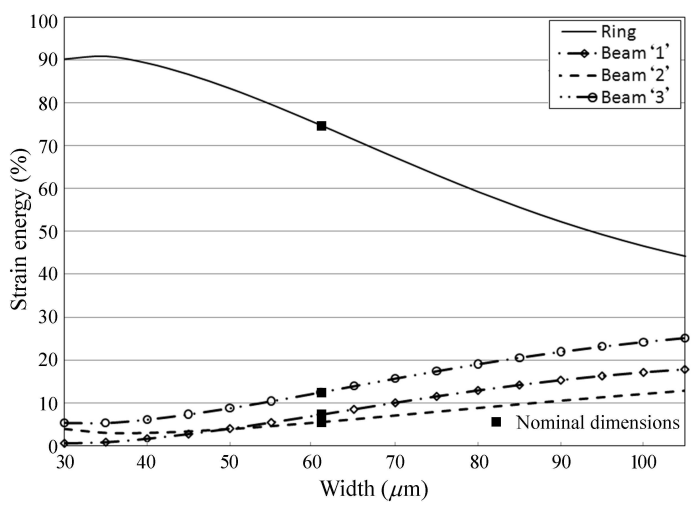

(b)

Figure 12: Kinetic energy (a) and strain energy (b) percentages in the different portions of the ring-based resonator, for the $2 \theta$ mode, as a function of the leg width.

shows how the kinetic and strain energies are distributed (as percentages) between the different parts of the structure as the leg width is changed. The legs are divided into three beam portions, numbered from the ring to the support, as explained in Section 4.2. The energy corresponding to a specific portion of a leg is combined with the energies for the same portion of all other legs.

From Figure 12(a), it is clear that the ring moves less when the leg is 
wider. One can see that the kinetic energy, which is mainly present within the ring for very thin legs is shifted to the legs themselves when the leg width is increased. The kinetic energy percentage of beam ' 3 ' remains close to zero, as expected because beam ' 3 ' is "clamped" at its end. The fact that the percentage of kinetic energy contained within the ring decreases when the leg width increases shows that the total energy stored decreases.

It can be seen in Figure 12(b), that the strain energy of the ring decreases when the leg width increases. The ring does not deform as much as for the case when the leg width is very thin. The strain energies in the leg portions increase, which can be explained because they have a larger width and also show a greater deformation. It is interesting to look at the energy of the third beam portion. As this increases, it is expected that the forces at the clamped end will also increase, meaning an increase in the amount of energy lost. This explains the $Q$-factor decrease shown in Figure 11.

\section{Conclusion and discussion}

Different models for the analysis of support loss, which all assume the support to be semi-infinite, have been compared. It has been found that they give $Q$-values in the same range of magnitude and exhibit similar trends when a parameter is varied. This has been shown for simple and for more complex resonator vibrations. However, the results obtained for the $Q$-factor show that the analytical model tends to overestimate $Q$, or underestimate the quantity of energy lost. The most plausible reason for this is the rigid attachment assumption. This assumption over-evaluates the stored energy 
(and therefore the associated $Q$-factor) as the vibrational energy is constrained to stay within the resonator and is thus artificially increased. In contrast, the PML and the infinite element methods model the resonator and support together. The support therefore presents a non-zero flexibility when analysing the resonator vibration. Consequently the energy stored within the resonator is smaller. It is also very difficult to predict and to model the real effect of the attachment on the resonator. Even a finite element analysis makes approximations in this region of high stress concentration.

It is worth noting that the semi-infinite support assumption is usually not realistic for practical MEMS devices. This is because the supporting structure to which the resonator is attached, often has dimensions of a similar order to the dimensions of the resonator itself. Furthermore, the supporting structure is often bonded rigidly to other materials (e.g. glass), which may also be attached to a metal base. Hence the support structure is finite in extent, with external boundaries that would in practice reflect some energy back into the resonator, reducing the likelihood that all energy propagating away from the resonator is lost. As the support typically has finite extent, its own end boundaries may also have an important effect on the motion of the resonator-support attachment point. In addition, internal structural damping will be present and attenuate waves propagating into the support. These factors are not taken into account in the semi-infinite model. In general, MEMS resonators have $Q$-factors that have high values which are sensitive to external factors. This characteristic helps to explain why the models presented yielded numerical values significantly different from experimental measurements. Despite this, it is expected that the value 
of the theoretical models is to predict the influence of different parameters on the $Q$-factor, so that resonators can be designed to have lower levels of damping.

The presented analytical method was applied to a practical ring-based resonator. In comparison to thermoelastic damping, support loss of the nominal design was found to be negligible. However, recent research on thermoelastic damping [38] indicates the possibility of designing resonators with reduced levels of thermoelastic damping, increasing the importance of support losses in resonator applications. In addition, other resonator designs, such as bulk-mode resonators $[39,40]$, have been shown to have very high $Q$-factors that are support loss limited.

A parametric study was performed to gain a better understanding of the factors that influence support loss for a ring-based resonator supported on legs. The main findings relating to resonator and support design were that thin, flexible legs produced a significant increase in $Q$-factor for the operating modes, whilst the $Q$-factor decreased if the leg frequencies coincided with the ring frequencies. In order to minimise the support loss, it is necessary to consider both the clamping force and energy contained in the resonator simultaneously.

Essential future work includes validation of the presented models for simple and complex-shaped resonators with experimental results. To increase the $Q$-factors, the use of blocking masses that reduce the transmission of vibration to the attachment points (suggested in [9]) is an interesting area of possible future research. Support loss models for out-of-plane vibrations are also essential for the next generation of multi-axis resonators [41]. 


\section{Funding acknowledgements}

This work was supported by Atlantic Inertial Systems.

\section{References}

[1] Lifshitz, R. and Roukes, M. L., Thermoelastic damping in microand nanomechanical systems. Phys. Rev. B, 2000, 61(8), 5600-5609.

[2] Yi, Y. B., Geometric effects on thermoelastic damping in MEMS resonators. J. Sound Vibr., 2008, 309, 588-599.

[3] Yasumura, K. Y., Stowe, T. D., Chow, E. M., Pfafman, T., Kenny, T. W., Stipe, B. C., and Rugar, D., Quality factors in micron- and submicron-thick cantilevers. J. Microelectromech. Syst., 2000, 9(1), 117-125.

[4] Chandorkar, S. A., Agarwal, M., Melamud, R., Candler, R. N., Goodson, K. E., and Kenny, R. W., Limits of quality factor in bulkmode micromechanical resonators, in Proc. IEEE MEMS 2008, Tucson, USA, 2008 pp 74-77.

[5] Tabrizian, R., Rais-Zadeh, M., and Ayazi, F., Effect of phonon interactions on limiting the f.Q product of micromechanical resonators, in Proc. Transducers 2009, Denver, USA, 2009 pp 2131-2134.

[6] Yang, J., Ono, T., and Esashi, M., Investigating surface stress: surface loss in ultrathin single-crystal silicon cantilevers. J. Vac. Sci. Technol., 2001, 19(2), 551-556. 
[7] Henry, J. A., Wang, Y., and Hines, M. A., Controlling energy dissipation and stability of micromechanical silicon resonators with selfassembled monolayers. Appl. Phys. Lett., 2004, 84(10), 1765-1767.

[8] Langdon, R. M., Resonator sensors - a review. J. Phys. E: Sci. Instrum., 1985, 18(2), 103-115.

[9] Haueis, M., Dual, J., and Buser, R., A mechanical isolation of a bending resonator. Sens. Actuators A, 2006, 128(2), 257-264.

[10] Judge, J. A., Photiadis, D. M., Vignola, J. F., Houston, B. H., and Jarzynski, J., Attachment loss of micromechanical and nanomechanical resonators in the limits of thick and thin support structures. J. Appl. Phys., 2007, 101(1), 013521.

[11] Park, Y. H. and Park, K. C., High-fidelity modeling of MEMS resonators - Part I: Anchor loss mechanisms through substrate. J. Microelectromech. Syst., 2004, 13(2), 238-247.

[12] Park, Y. H. and Park, K. C., High-fidelity modeling of MEMS resonators - Part II: Coupled beam-substrate dynamics and validation. $J$. Microelectromech. Syst., 2004, 13(2), 248-257.

[13] Hao, Z., Erbil, A., and Ayazi, F., An analytical model for support loss in micromachined beam resonators with in-plane flexural vibrations. Sens. Actuators A, 2003, 109, 156-164. 
[14] Miller, G. F. and Pursey, H., The field and radiation impedance of mechanical radiators on the free surface of a semi-infinite isotropic solid. P. R. Soc. Lond., 1954, 223, 521-541.

[15] Jimbo, Y. and Itao, K., Energy loss of a cantilever vibrator. J. Horolog. Inst. Jpn., 1968, 47, 1-15 (in Japanese).

[16] Cross, M. C. and Lifshitz, R., Elastic wave transmission at an abrupt junction in a thin plate with application to heat transport and vibrations in mesoscopic systems. Phys. Rev. B, 2001, 64(8), 1-22.

[17] Hao, Z. and Xu, Y., Vibration displacement on substrate due to timeharmonic stress sources from a micromechanical resonator. J. Sound Vibr., 2009, 322, 196-215.

[18] Chouvion, B., Vibration transmission and support loss in MEMS sensors, Ph.D. thesis, Univ. Nottingham, UK, 2010.

[19] Graff, K. F., Wave Motion in Elastic Solids, Ohio State University Press, 1975.

[20] Chouvion, B., Fox, C. H. J., McWilliam, S., and Popov, A. A., In-plane free vibration analysis of combined ring-beam structural systems by wave propagation. J. Sound Vibr., 2010, 329(24), 5087-5104.

[21] Chouvion, B., Popov, A. A., McWilliam, S., and Fox, C. H. J., Vibration modelling of complex waveguide structures. Comput. Struct., 2011, 89, 1253-1263. 
[22] Engquist, B. and Majda, A., Absorbing boundary conditions for the numerical simulation of waves. Math. Comput., 1977, 31(139), 629-651.

[23] Givoli, D., Patlashenko, I., and Keller, J. B., Discrete Dirichletto-Neumann maps for unbounded domains. Comput. Method. Appl. M., 1998, 164, 173-185.

[24] Astley, R. J., Infinite elements for wave problems: a review of current formulations and an assessment of accuracy. Int. J. Numer. Meth. Eng., 2000, 49(7), 951-976.

[25] Cheng, A. H. D. and Cheng, D. T., Heritage and early history of the boundary element method. Eng. Anal. Bound. Elem., 2005, 29(3), 268-302.

[26] Bérenger, J. P., A perfectly matched layer for the absorption of electromagnetic waves. J. Comp. Phys., 1994, 114, 185-200.

[27] Kosloff, R. and Kosloff, D., Absorbing boundaries for wave propagation problems. J. Comp. Phys., 1986, 63(2), 363-376.

[28] Bindel, D. S. and Govindjee, S., Elastic PMLs for resonator anchor loss simulation. Int. J. Numer. Meth. Eng., 2005, 64(6), 789-818.

[29] Bindel, D., HiQLab, available online at http://www.cims.nyu.edu/ dbindel/, accessed in Jan. 2010.

[30] Koyama, T., Efficient evaluation of damping in resonant MEMS, Ph.D. thesis, Univ. California, Berkeley, USA, 2008. 
[31] Zienkiewicz, O. C., Emson, C., and Bettess, P., A novel boundary infinite element. Int. J. Numer. Meth. Eng., 1983, 19(3), 393-404.

[32] Lysmer, J. and Kuhlemeyer, R. L., Finite dynamic model for infinite media. J. Eng. Mech. div.-ASCE, 1969, 95(4), 859-877.

[33] Dassault Systèmes, Abaqus Online documentation, Abaqus Theory manual, Version 6.7, 2007.

[34] Grigg, H. T. D., Towards a parametrically pumped xylophone microbar magnetometer: design optimisation of xylophone bar resonator, Proc. IMAPS DPC, 2011, (In Press).

[35] Wong, S. J., Thermoelastic damping in MEMS ring resonators, Ph.D. thesis, Univ. Nottingham, UK, 2005.

[36] Wu, C. M. and Lundberg, B., Reflection and transmission of the energy of harmonic elastic waves in a bent bar. J. Sound Vibr., 1996, $\mathbf{1 9 0}(4), 645-659$.

[37] Hao, Z. and Ayazi, F., Support loss in micromechanical disk resonators, in Proc. IEEE MEMS 2005, Miami, 2005 pp 137-141.

[38] Duwel, A., Candler, R. N., Kenny, T. W., and Varghese, M., Engineering MEMS resonators with low thermoelastic damping. J. Microelectromech. Syst., 2006, 15(6), 1437-1445.

[39] Hao, Z., Pourkamali, S., and Ayazi, F., VHF single-crystal silicon elliptic bulk-mode capacitive disk resonators-part I: design and modeling. J. Microelectromech. Syst., 2004, 13(6), 1043-1053. 
[40] Abdelmoneum, M. A., Demirci, M. U., and Nguyen, C. T.C., Stemless wine-glass-mode disk micromechanical resonators, in Proc. IEEE MEMS 2003, Kyoto, Japan, 2003 pp 698-701.

[41] Fox, C., McWilliam, S., Eley, R., and Fell, C., Development of multi-axis rate sensors based on vibrating silicon ring structures, in Proc. RTO SET Symp. "Emerging Military Capabilities Enabled by Advances in Navigation Sensors", Istanbul, Turkey, 2002 pp 13.1-13.12. 


\section{List of Figures}

1 Semi-infinite thin plate with excitation sources. . . . . . . 5

2 Linear attenuation function used in the PML method for a one-dimensional wave. . . . . . . . . . . . . . . 11

3 Nodal variable position for a one-dimensional infinite element in $\mathrm{ABAQUS}^{\mathrm{TM}} \ldots \ldots \ldots \ldots \ldots \ldots \ldots \ldots$

4 Convergence analysis. $Q$-factor function of $R / \lambda$ calculated for different mesh densities. . . . . . . . . . . . . . 15

5 Cantilever beam case. $Q$-factor calculated using the three different methods as a function of the length/width ratio. . . 17

6 Two-beam case. $Q$-factor calculated using the different methods as a function of the length/width ratio of beam ' 2 '. The system studied is shown in the inset. Schematic representations of the mode shapes, for the shortest and longest beam '2' considered, are also illustrated. . . . . . . . . . . . . 19

7 Photograph of a ring-based rate sensor on its silicon wafer (courtesy AIS), and schematic representation of the resonator. 21

$82 \theta$ and $3 \theta$ mode shapes of the ring-based resonator with nominal dimensions. . . . . . . . . . . . . . . . . . . 22

$9 \quad Q$-factors of the $2 \theta$ mode for different values of $L_{1}$ and $L_{3} \ldots .25$

10 Deformed shape of the $2 \theta$ mode for a long beam ' 1 ' and a short beam '3' (a), and for a short beam '1' and a short beam

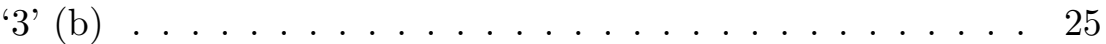


$112 \theta$ frequencies $(\mathrm{Hz})$ and the associated $Q$-factors function of the leg width. . . . . . . . . . . . . . . . . 28

12 Kinetic energy (a) and strain energy (b) percentages in the different portions of the ring-based resonator, for the $2 \theta$ mode, as a function of the leg width. . . . . . . . . . . . . 29 